\title{
Leaky Wave Array in Full Planar Substrate with EBG-Based Wave Guiding Channel
}

\author{
Linghui Kong (iD, ${ }^{1}$ Sen Yan $\left(\mathbb{D},{ }^{2}\right.$ Vladimir Volskiy, ${ }^{3}$ Binke Huang, ${ }^{2}$ and Guy A. E Vandenbosch ${ }^{3}$ \\ ${ }^{1}$ School of Electronics and Information Engineering, Soochow University, Suzhou 215006, China \\ ${ }^{2}$ School of Electronics and Information Engineering, Xi'an Jiaotong University, Xi'an 710049, China \\ ${ }^{3}$ The ESAT - Telemic Research Division, KU Leuven, Leuven 3001, Belgium \\ Correspondence should be addressed to Sen Yan; sen.yan@xjtu.edu.cn
}

Received 19 January 2021; Accepted 10 May 2021; Published 25 May 2021

Academic Editor: Giuseppe Castaldi

Copyright ( 92021 Linghui Kong et al. This is an open access article distributed under the Creative Commons Attribution License, which permits unrestricted use, distribution, and reproduction in any medium, provided the original work is properly cited.

\begin{abstract}
A new type of wave guiding structure is proposed is this paper. The guiding channel is developed on the full planar dielectric substrate and aligned with electromagnetic bandgap (EBG) units. Since the bandgap of these mushroom-like units is calculated with a coverage of the channel working band, these units are of great importance on ensuring the transmission efficiency and eliminating the coupling effect between channels. Then, this wave guiding structure is extended to the design of a six-element leaky wave antenna array with a complete size of $25.6 \mathrm{~mm} \times 80.6 \mathrm{~mm}$, which is working at $\mathrm{Ku}$ band from $12.0 \mathrm{GHz}$ to $12.8 \mathrm{GHz}$ and achieving a bandwidth of about $0.8 \mathrm{GHz}$, a gain of $11.36 \mathrm{dBi}$, and an efficiency of ca. $86.7 \%$ at $12.0 \mathrm{GHz}$. Within the working frequency band, this antenna topology achieves a frequency-dependent beam scanning in the forward directions, and it offers a potential for radar application on road speed detecting with low costs.
\end{abstract}

\section{Introduction}

Nowadays, the development of CMOS technology enables circuits to operate in higher and higher frequency ranges, such as the mm-wave frequency band of $30 \mathrm{GHz}$ to $300 \mathrm{GHz}$. By making use of carrier frequencies in this frequency domain, wideband communication systems can benefit from the large bandwidth and high data rate. However, one of today's challenges is to transmit these wave carriers over a limited distance from several centimeters to meters to provide a multi-gigabit communication link [1]. The traditional planar strip lines such as microstrip or coplanar waveguide will be seriously suffered from both dielectric and conductor losses. In contrast, dielectric transmission media always enjoy with the low material loss which makes it an ideal candidate for a low-loss, cost-friendly, and light-weight wave-guiding channel [2].

Several decades ago, dielectric transmission lines such as the Goubau line and dielectric strips were known for their very low transmission loss but at the expense of a complicated mechanical mounting structure and a rather large extension of the fields into free space [3, 4]. Later, grounded dielectric rods and rectangular strips (the so-called image guide) were investigated, also showing a quite low attenuation [5, 6]. A nonradiative dielectric (NRD) waveguide is proposed to work at $94 \mathrm{GHz}$ band with the air holes to lower the dielectric permittivity [7]. The substrate integrated waveguide (SIW) has gained a wide popularity in millimeter wave range, but it still faces with the conductive losses as enclosed by metal shielding parts [8]. A new version of the image guide, called the Substrate Integrated Image Guide (SIIG), was first proposed in [9]. With a thinner insulator layer, it offers a new type of dielectric transmission line for multiple layer applications. A comparison of the propagation characteristics of these different transmission lines is presented in Table 1.

To further extend the range of applications, wave guiding channels can be realized in full planar substrates with the electromagnetic bandgap (EBG) units [10]. EBG structures are categorized as a type of metamaterial with artificial or nonartificial periodic units. With the adequate design parameters, materials, and shapes, they can be used to control 
TABLE 1: Comparison of different structures.

\begin{tabular}{lcccc}
\hline Trans. lines & RDW [4] & NRG [7] & SIW [8] & SIIG [9] \\
\hline Bandwidth $(\mathrm{GHz})$ & $10-18$ & $92 \sim 99.2$ & $38 \sim 59$ & $80 \sim 95$ \\
S21 $(\mathrm{dB})$ & $<-2$ & $-5.4 \sim-3$ & $-1.2 \sim-1.0$ & $<-3$ \\
S11 $(\mathrm{dB})$ & $<-13$ & $<-10$ & $<-15$ & $/$ \\
Trans. loss $(\mathrm{dB} / \mathrm{mm})$ & $0.019 \sim 0.04$ & $/$ & TE $_{11}$ & 0.025 \\
Trans. mode & $\mathrm{HE}_{11}$ & LSE $_{11}$ & $\mathrm{E}^{\mathrm{y}}$ & \\
Dimension $(\mathrm{mm})$ & $10 \times 25 \times 10$ & $0.635 \times 2.84 \times 26.3$ & $0.381 \times 2.6 \times 4$ & $0.525 \times 11.49 \times 40$ \\
Material & RO3010 & Alumina & Alumina & TMM10i, silicon \\
Mode transition & WR-62 & WR-10 & CPW & WR-10 \\
\hline
\end{tabular}

and modulate the propagation of electromagnetic waves in the planar substrate. In the design of planar antennas, this can be applied to suppress side leakages and avoid the mutual couplings between channels [11].

Leaky wave antennas (LWAs) can achieve a high directivity with a simple structure without the need for a complex and costly feeding network [12], and the capability of beam scanning with frequency changes [13]. Radiations from LWAs are generated by the continuous or periodic power leakage from transmission lines into the exterior region. The first LWA was proposed in the 1940s in the form of a slotted rectangular waveguide [14]. Recent developments are focusing on planar LWAs to improve the scanning range and overcome the 'stop band' problem in the broadside direction $[15,16]$.

In this paper, an innovating $1 \mathrm{D}$ leaky wave antenna topology is proposed, which combines several aspects:

The structure is based on a fully planar and very lowprofile substrate

A dielectric waveguide is formed based on this grounded substrate in combination with mushroom EBG units along the guiding channel

This waveguide is used as the feeding mechanism for a leaky wave planar array [17]

Furthermore, a dedicated CPW to SIW transition structure with a $\mathrm{V}$ slot is adopted to achieve a smooth mode conversion to the guiding channel

As far as the authors can see, this is the first structure described in literature combining all these features. This topology offers significant advantages in integration, fabrication, and a broad working band when compared with the conventional transmission lines.

The content is organized as follows. First, the EBG-based dielectric waveguide is described and the propagation efficiency is verified. Then, a CPW to SIW feeding topology is employed to excite the working mode inside the waveguide. Finally, a Kuband periodic leaky wave antenna, which is made of six metal strips on top of the guiding channel, is proposed. The measured return loss, far field pattern, and realized gain are compared with simulations, and a good agreement is observed.

\section{Wave Guiding Structure}

The complete topology of the proposed transmission medium is shown in Figure 1. It is composed of three parts: the middle guiding channel, the feeding part, and the EBG units.

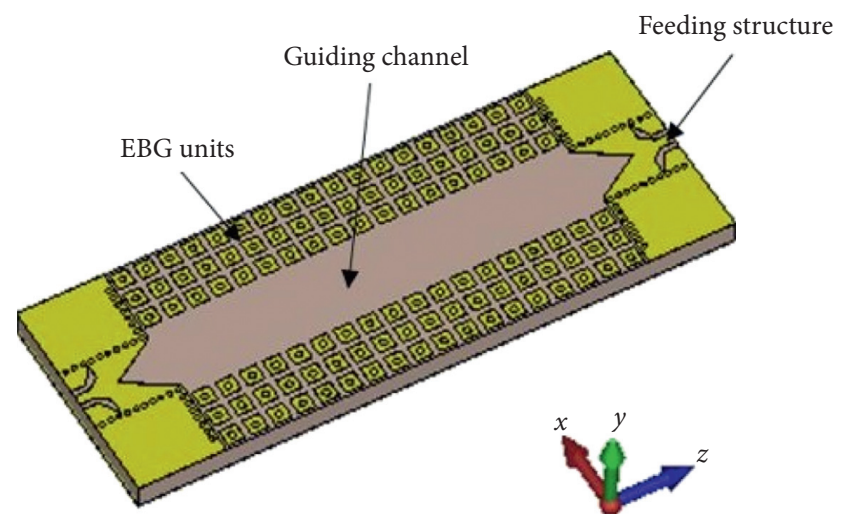

FIgURE 1: EBG-based dielectric waveguide.

While the middle channel is designed to work in the required frequency band, the EBG units are optimized to obtain the stopband with a coverage of the frequency band. In this way, the side leakages and channel couplings can be suppressed to achieve a board-level dielectric interconnector design. In this work, all the simulations are performed within CST Microwave Studio.

2.1. Dielectric Guiding Channel. The initial dielectric image guide (DIG), as shown in Figure 2, is designed with a piece of low-loss high permittivity Rogers RT 6010 substrate (permittivity 11.4 , loss tangent 0.0023 , and thickness $2.54 \mathrm{~mm}$ ). The high permittivity material is chosen to have a higher field concentration within the waveguide core. The degenerate $\mathrm{E}^{\mathrm{x}}{ }_{11}$ mode with a parallel electric field can be avoided by the metal ground. Hence, only the $E_{11}^{\mathrm{y}}$ mode (Figure 3(a)) is left as the dominant mode and to propagate inside the channel (Figures 3(b), and Figure 4 gives the insertion losses of the first two modes. It can be seen that a single mode transmission has been achieved below $14.5 \mathrm{GHz}$.

2.2. Eigenmode Analysis of EBG Unit. In many cases, the eigenmode analysis of EBG structures is based on the Bloch-Floquet theorem, which describes the wave propagation in an infinite medium consisting of periodic unit cells. The relationship between the propagation constant and frequency can be given by the dispersion diagram.

For a two-dimensional periodical structure, the dispersion diagram can be given by starting from $\Gamma(0,0)$, going 


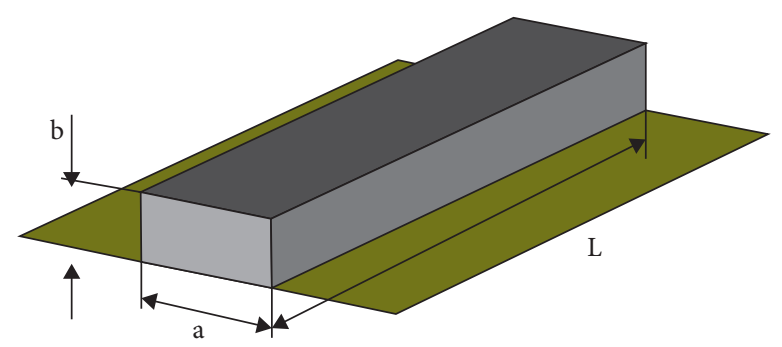

Figure 2: Grounded image guide $(a=8.8 \mathrm{~mm}, b=2.54 \mathrm{~mm}$, and $L=30 \mathrm{~mm})$.
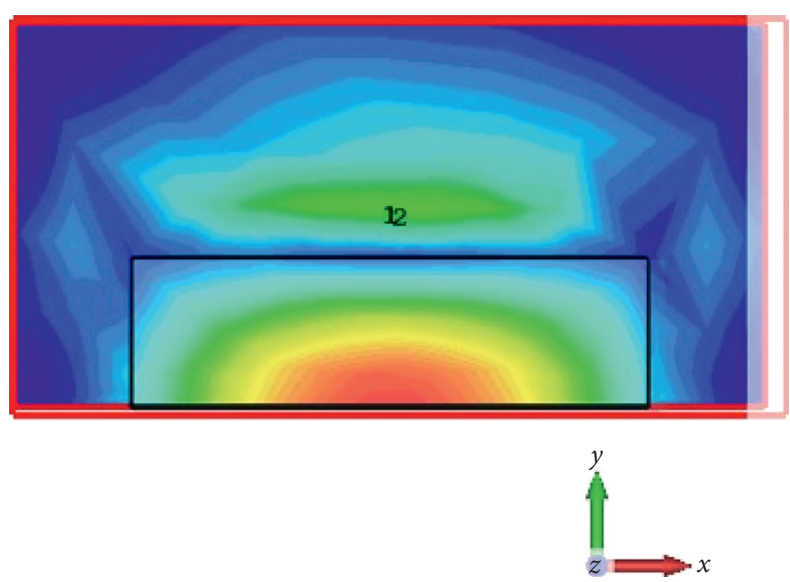

(a)

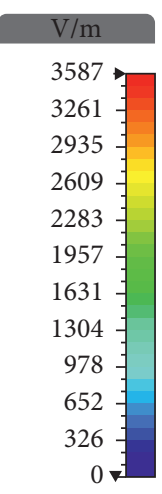

FIGURE 3: $\mathrm{E}_{11}^{\mathrm{y}}$ mode of the dielectric image guide: (a) port mode field and (b) propagation mode field.
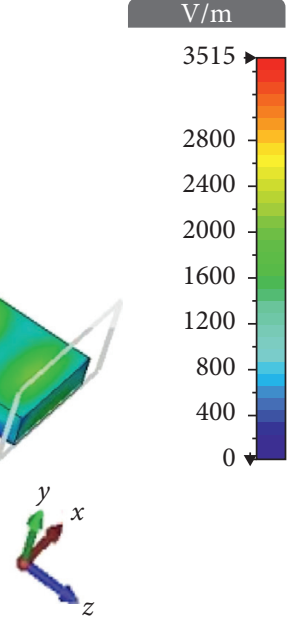

(b)

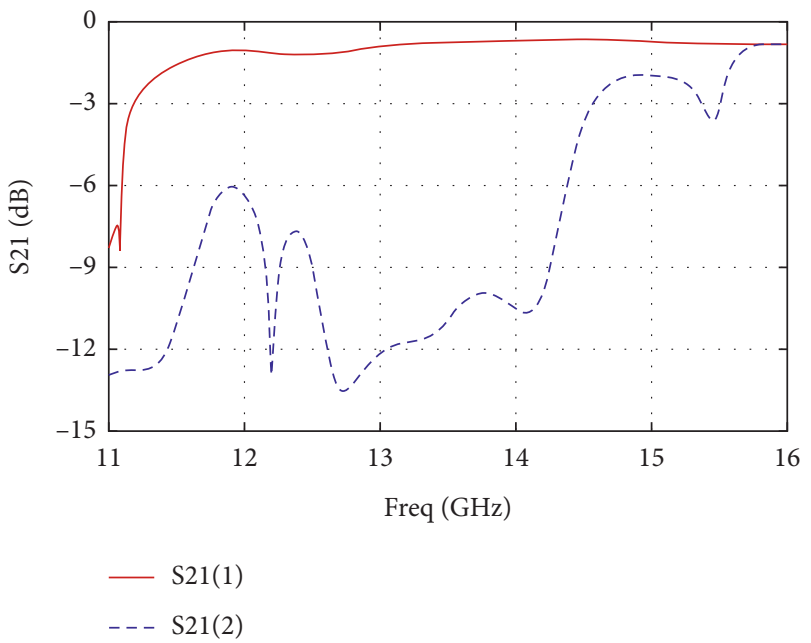

FIgURE 4: Transmission efficiency of the first two modes (with (1) and (2) represent $E_{11}^{y}$ and $E_{21}^{y}$, respectively) of the grounded DIG.

to $X(0, \pi)$, then to $M(\pi, \pi)$ and back to $\Gamma$, as indicated in Figure 5(a). In this work, a mushroom-like unit is used (also see in Figure 5(a)), consisting of one central square patch with metal vias connected to the ground.

In order to mimic the periodicity of the structure, the boundary conditions at the side walls are defined as periodic boundaries. As the eigenmode solver in CST does not support open boundary conditions, both the top and bottom sides are defined as electric conductors, see Figure 5(b). Also, consider that the fields are mainly concentrated inside the substrate, and just above the substrate, air interface, a proper height of the air layer, i.e., more than ten times the substrate 


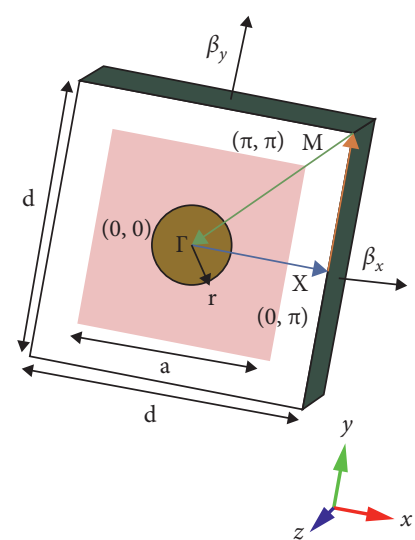

(a)

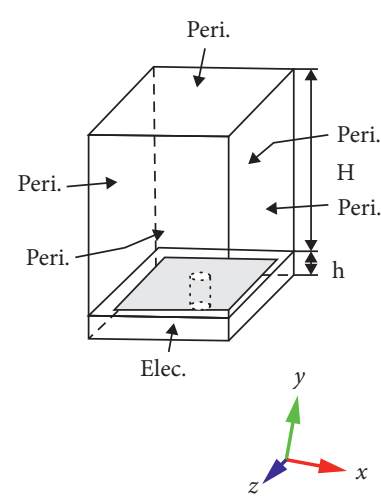

(b)

Figure 5: (a) Mushroom-type square EBG unit of size $d=2.8 \mathrm{~mm}$, consisting of a square patch with size a $=2.0 \mathrm{~mm}$, connected in the center to the ground by a via with radius $r=0.3 \mathrm{~mm}$. The points defining the path in the Brillouin zone are also indicated. (b) Boundary conditions in the simulations.

thickness, yields the correct field distribution of the eigenmodes.

The dispersion diagram is plotted in Figure 6. As discussed, it incorporates three intervals ( $\Gamma$ to $X, X$ to $M$, and $M$ to $\Gamma$ ) to describe all possible propagation directions in the $x y$ plane. In each direction, the phase changes are swept from 0 to $\pi$, and the cutoff frequency for each eigenmode is calculated.

It is known that the mushroom-like EBG structure allows the propagation of TM waves at low frequencies and TE waves at high frequencies [18]. The first mode is thus a TM mode, which starts as a forward propagating TEM mode at very low frequency and low wavenumber and changes into a forward TM surface wave. Modes 2, 3, and 4 are hybrid modes that start as TE waves at a very low wavenumber and transform into TM waves at high frequencies.

Figure 7 presents the electric field distribution in the $y z$ plane of the first four eigenmodes. We can see that mode 2 is orthogonal to the transmission mode inside the waveguide, and mode 2 will not be excited and can be neglected from considerations. Meanwhile, since the transmission mode inside the dielectric image guide is a slow wave with the phase speed slower that the light, the frequency band gap should also below the frequency line of light [19], as seen in Figure 6. Therefore, the band gap for this periodic structure is ranging from about $6.5 \mathrm{GHz}$ to $15.0 \mathrm{GHz}$.

2.3. Feeding Structure. In order to achieve a smooth mode conversion from the feeding structure to the guiding channel, a CPW to SIW (substrate integrated waveguide) transition with a $\mathrm{V}$ slot is used to excite the transmission mode inside the guiding channel (Figure 8). The complete feeding structure is obtained by a step-by-step design procedure and finally assembles each part together. The listed parameters are optimized to ensure that the different parts can satisfy the working frequency band. Among them, the dimensions of the tapered CPW and V-slot play the key roles on determining the frequency range and transmission efficiency. Besides, this planar structure is important for the interconnections between MMIC chips and coaxial cables. The tapered CPW feed line with ground is employed to match with SIW and results in a wide band transition $[20,21]$. The fundamental mode inside the waveguide is similar to $\mathrm{TE}_{10}$ mode, while the cutoff frequency can be calculated by the sectional dimensions and the design parameters of the metal vias as follows [22]:

$$
f_{c}=\frac{c}{2 \sqrt{\varepsilon_{r}}\left(a_{s}-d^{2} / 0.95 p\right)},
$$

and the equivalent wavelength of SIW is obtained by

$$
\lambda_{g}=\frac{2 \pi}{\sqrt{\varepsilon_{r}(2 \pi f)^{2} /\left(\pi / a_{s}\right)^{2}}},
$$

where $\varepsilon_{r}$ is the relative permittivity of the substrate material, $a_{s}$ refers to the distance between the two rows of metal vias, $d$ is the diameter of the vias, $p$ represents the periodical distance between vias, and $c$ is the speed of light. With the design parameters listed in Figure 8, we can get $f_{c T E_{10}}=$ $6.61 \mathrm{GHz}$ and $f_{c T E_{20}}=13.22 \mathrm{GHz}$. In the upper frequency limit of $12.8 \mathrm{GHz}$, the equivalent wavelength is calculated with $\lambda_{g}=7.92 \mathrm{~mm}>a_{s}$. Therefore, these computed results show that only the dominant mode can be propagated inside the SIW within the operation frequency range of 12.0 to $12.8 \mathrm{GHz}$.

In Figure 9, the electric field distributions in the different parts of the structure are presented. The transmitted $\mathrm{TE}_{10}$ mode within SIW is well matched with the dominant $E_{11}^{y}$ mode in DIG, delivering a smooth mode conversion. The simulated $S$ parameters (Figure 10) show that a reasonable transmission efficiency is achieved in the desired frequency range. In order to illustrate the influence from the EBG units, the reflection and transmission parameters are also given in this figure for the original dielectric waveguide without these units in comparison. It is clearly seen that the presence of these mushroom units can generate a wave guiding channel and, moreover, eliminate a series of resonances in the 


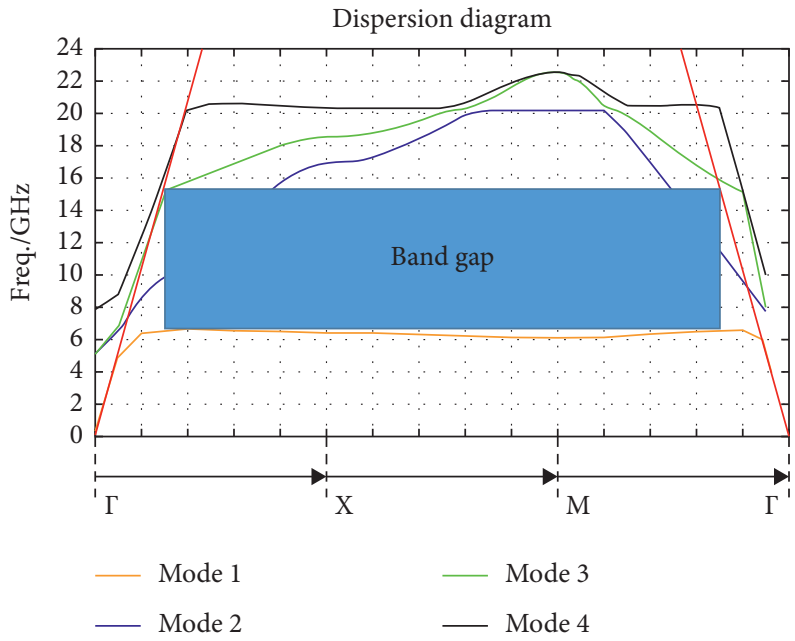

FIgure 6: Dispersion diagram of the EBG unit (with red lines for the light cutoff frequency).

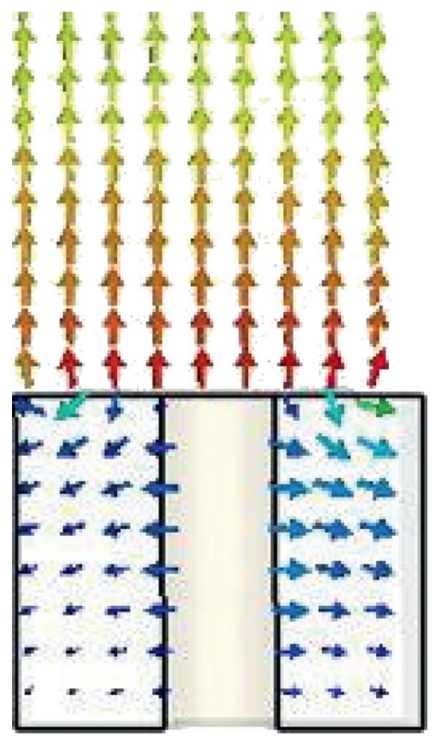

(a)

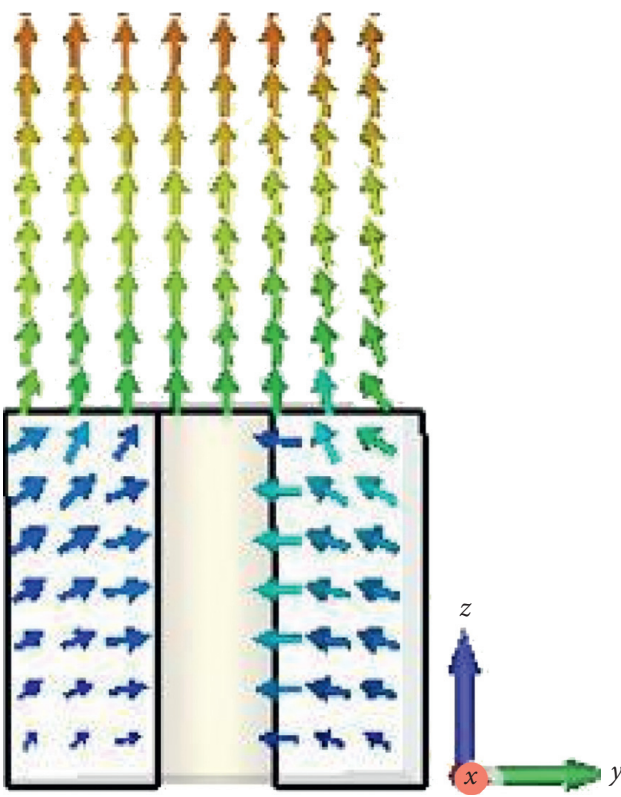

(c)

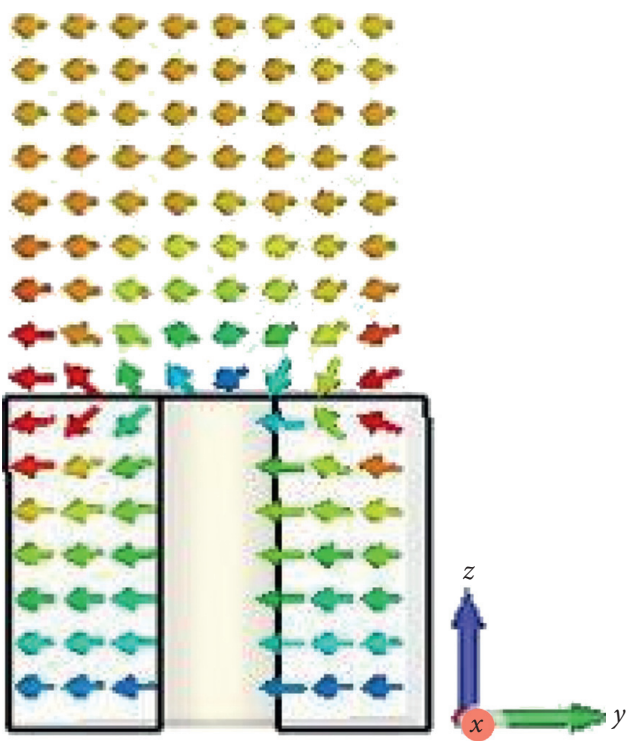

(b)

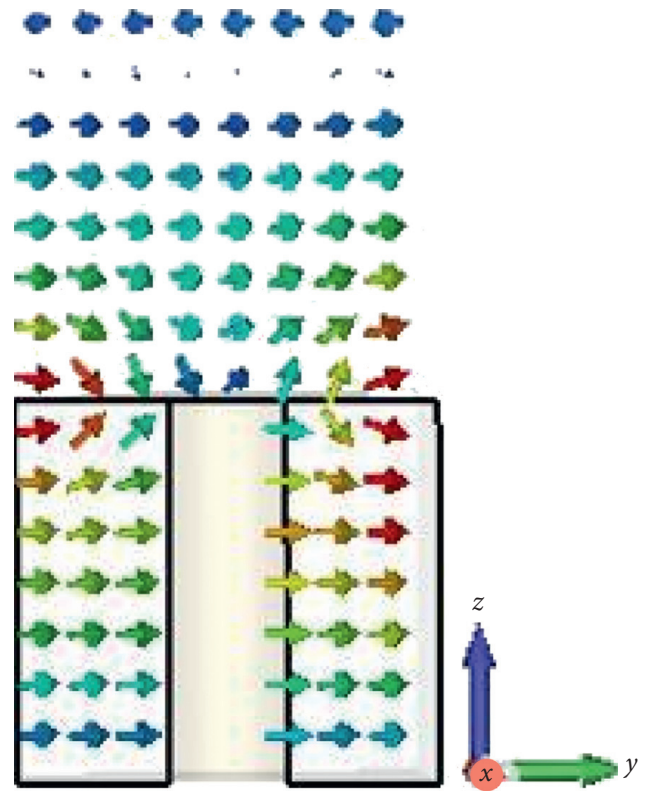

(d)

Figure 7: Sectional view of electric field distribution of the four eigenmode in $y z$ plane. (a) Mode 1. (b) Mode 2. (c) Mode 3. (d) Mode 4. 


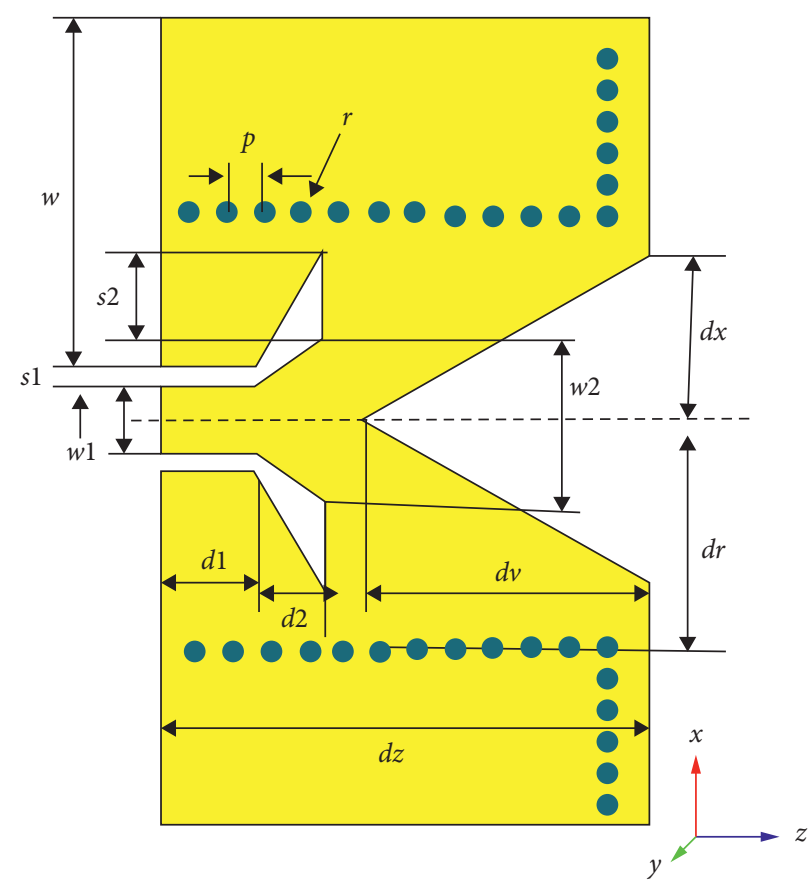

Figure 8: Feeding structure $(w=11.35, w 1=1.3, w 2=3.2, s 1=0.8, s 2=1.8, d 1=2, d 2=1.4, \mathrm{dv}=5.6, \mathrm{dx}=3.4, \mathrm{dz}=10.4, \mathrm{dr}=3.6, p=0.8, \mathrm{and}$ $r=0.3$. Unit: $\mathrm{mm}$ ).

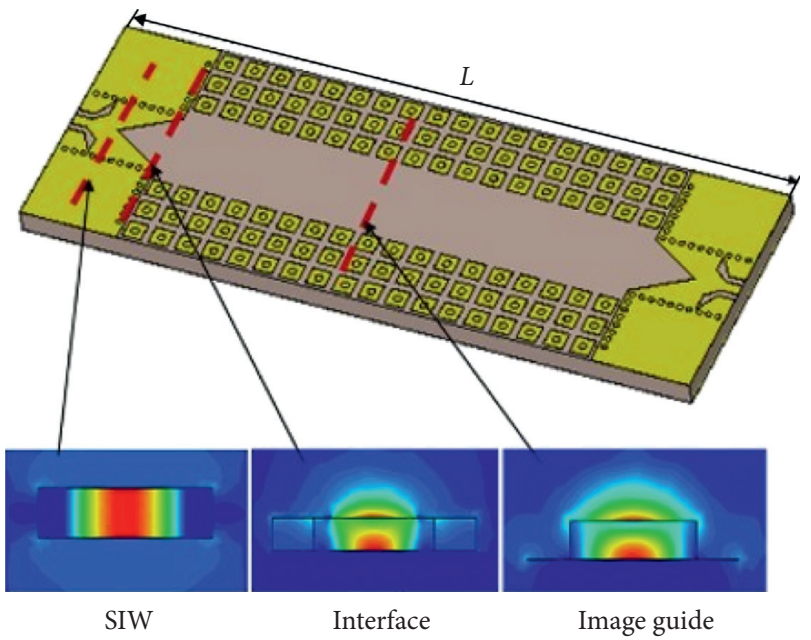

FIGURE 9: Mode conversion from the feeding structure to guiding channel. The length of the structure $L=71.2 \mathrm{~mm}$.

structure, giving it a much more stable $S_{21}$ in the operational band than the original dielectric waveguide. Although there are some ripples appeared in the $S_{11}$ performance, it still keeps below $-10 \mathrm{~dB}$ at the target frequency range $(12.0$ - $12.8 \mathrm{GHz}$ ) even when compared with the structure without EBG units.

\section{Leaky Wave Antenna Array}

Based on the proposed dielectric waveguide, a Ku-band periodic leaky wave antenna array is designed and shown in Figure 11. It contains six metal strips in the top interface.

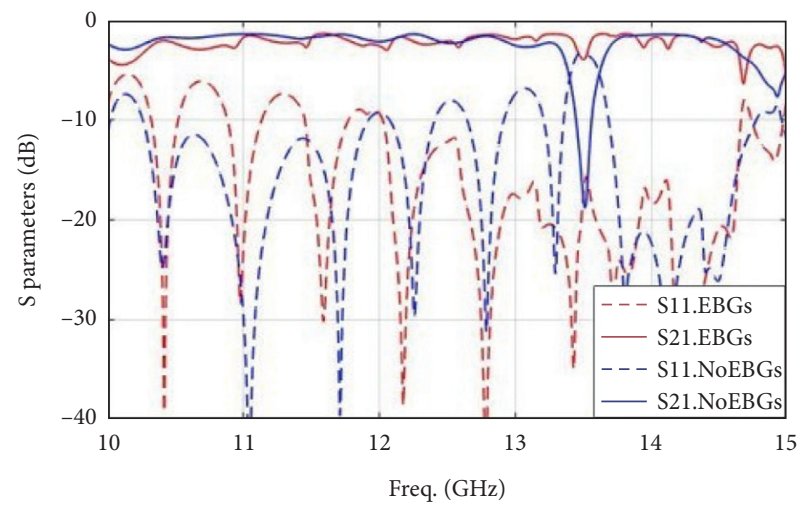

FIgure 10: Comparison of the simulated reflection and transmission parameters of the waveguide realized with the EBG units and the original dielectric waveguide.

The fabricated prototype and the measurement setup in the anechoic chamber are shown in Figure 12. The return loss is measured with a network analyzer and agrees satisfactory with simulations (Figure 13). The reflection coefficient is below $-10 \mathrm{~dB}$ in the frequency range of $12.0-12.8 \mathrm{GHz}$, which proves that this antenna prototype can work efficiently at this band. Also, the simulation yields that, at $12.5 \mathrm{GHz}$, about $79.9 \%$ of the total energy is radiated.

In Figure 14, the polar radiation pattern is plotted at different frequencies, $12.0 \mathrm{GHz}, 12.5 \mathrm{GHz}$, and $12.8 \mathrm{GHz}$, with main beam directions of $82^{\circ}, 65^{\circ}$, and $52^{\circ}$, respectively, indicating a scanning in the forward quadrant. The main lobe magnitude at these frequencies is $11.36 \mathrm{dBi}, 11.97 \mathrm{dBi}$, and $11.71 \mathrm{dBi}$, respectively. 


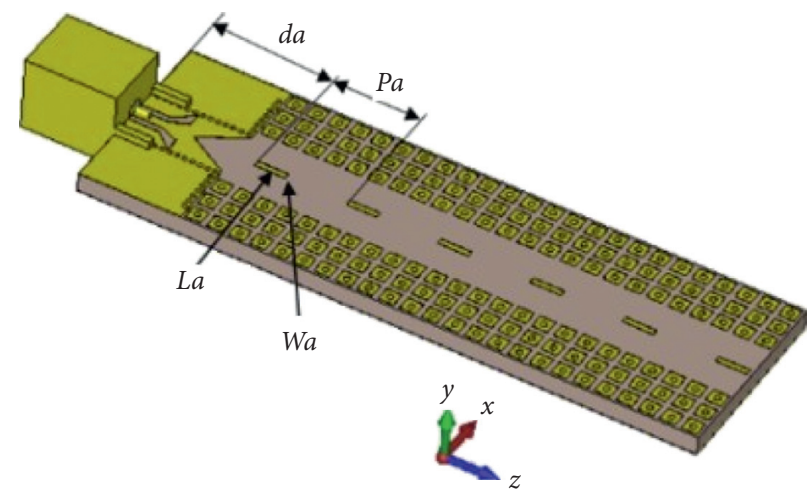

Figure 11: Configuration of the proposed $\mathrm{Ku}$-band periodic leaky wave antenna array $(\mathrm{La}=4, \mathrm{Wa}=0.8, \mathrm{~Pa}=12, \mathrm{da}=13.4$, unit: $\mathrm{mm})$.

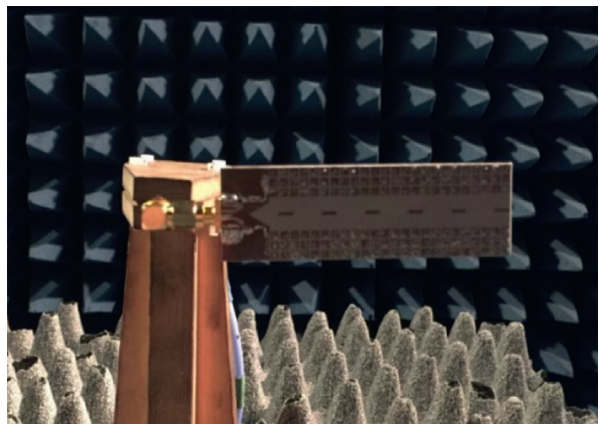

Figure 12: Far field measurement setup with the fabricated prototype.

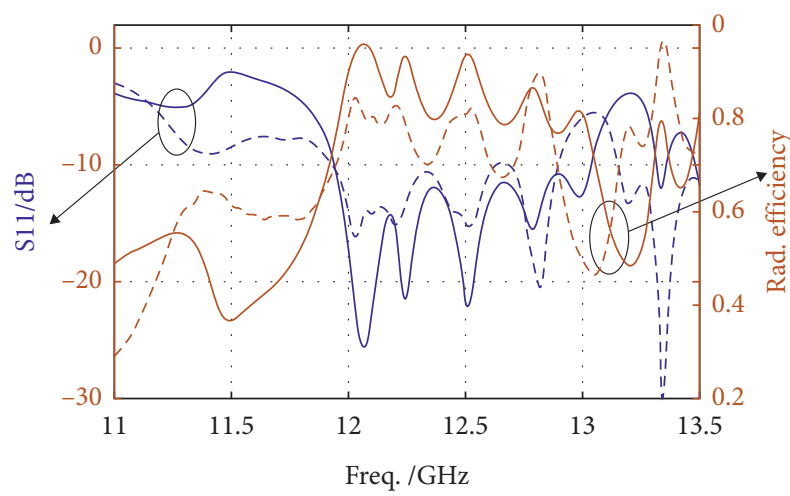

FIGURE 13: Comparisons of $S$ parameters and the radiation efficiency, with red lines for $S$ parameters and blue ones for the radiation efficiency, solid lines represent the simulated results, and the dashed lines represent the measurements.

In Figure 15, the normalized far field patterns at 12.0 $\mathrm{GHz}, 12.5 \mathrm{GHz}$, and $12.8 \mathrm{GHz}$ are plotted in XOY and YOZ planes with both co- and cross-polarization components, respectively. In the $X O Z$ plane, since the main beam is changed from the normal direction to the forward sides, as seen in Figure 14, the co-polarizations also decrease a lot at the frequency of $12.5 \mathrm{GHz}$ and 12.8 GHz. Even though, there is still a good agreement between the measured and simulated results on co-polarized components. As the measurements are easily

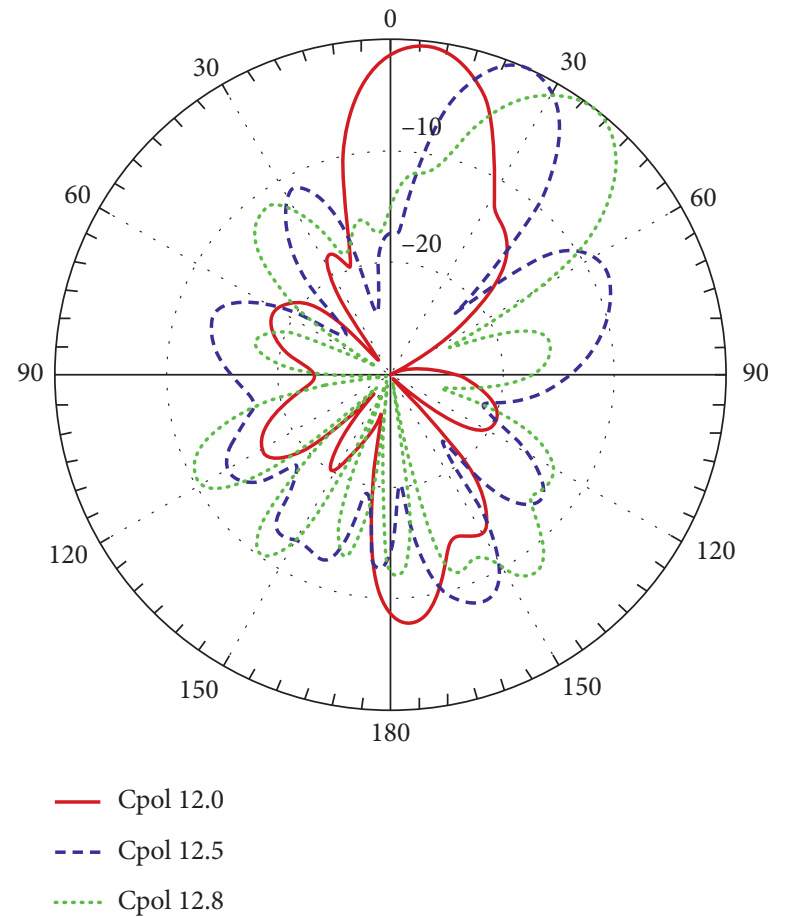

Figure 14: Simulated radiation pattern in E-plane (yOz plane); Cpol refers to the co-polarization $\left(\mathrm{E}_{\theta}\right)$ component.

influenced by the small radiation from the feeding cable at a quite low magnitude, a large discrepancy is observed in Figure 12. However, a similar trend can be seen 

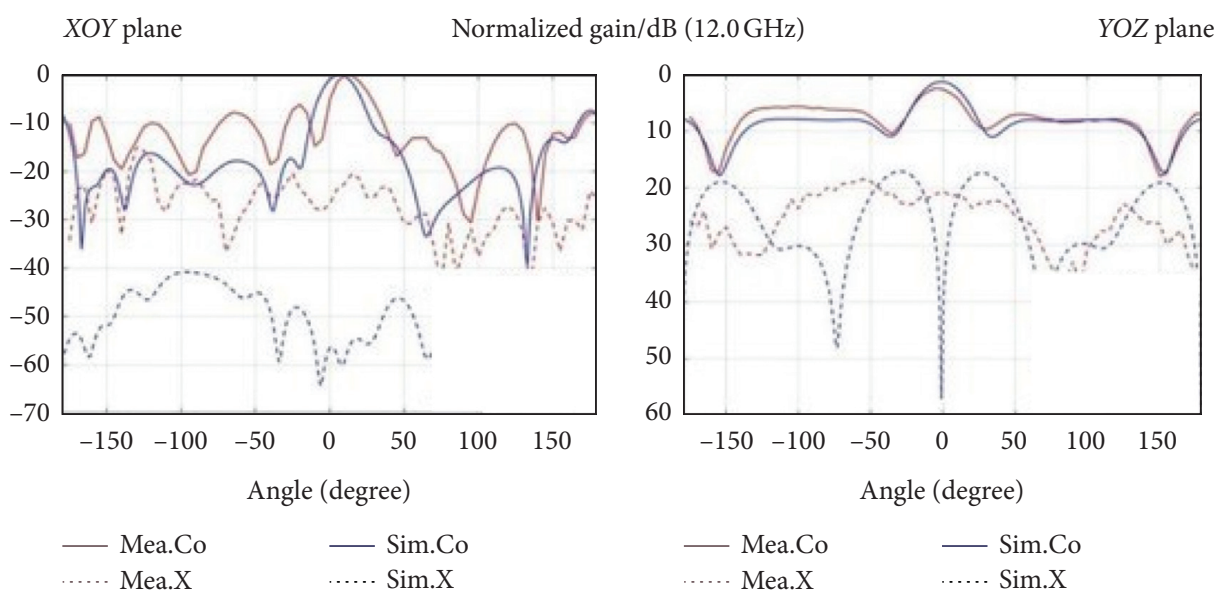

Normalized gain/dB $(12.5 \mathrm{GHz})$
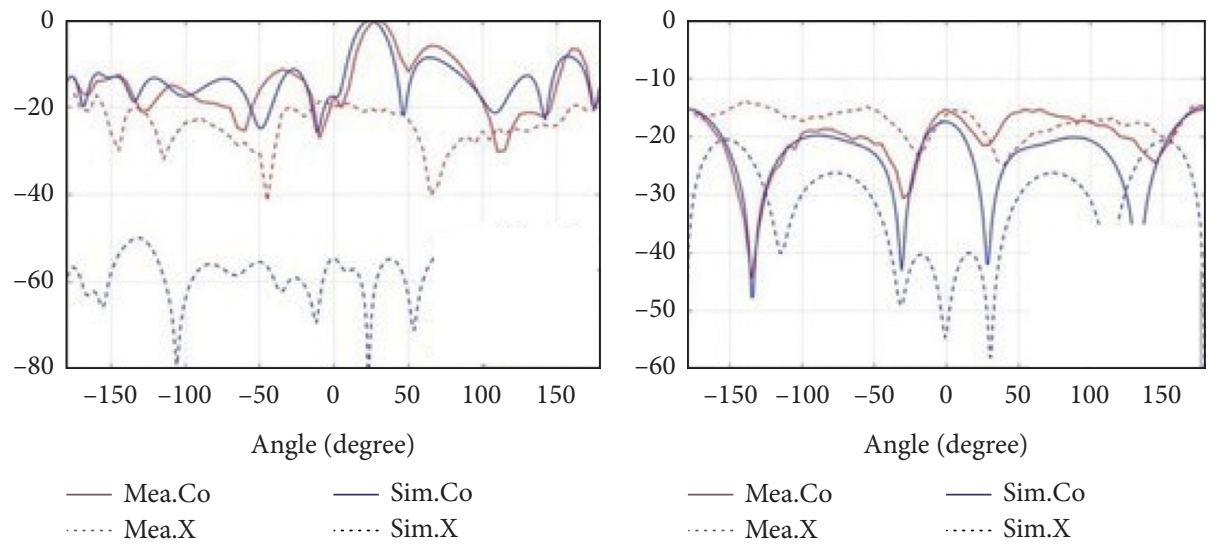

Normalized gain/dB $(12.8 \mathrm{GHz})$
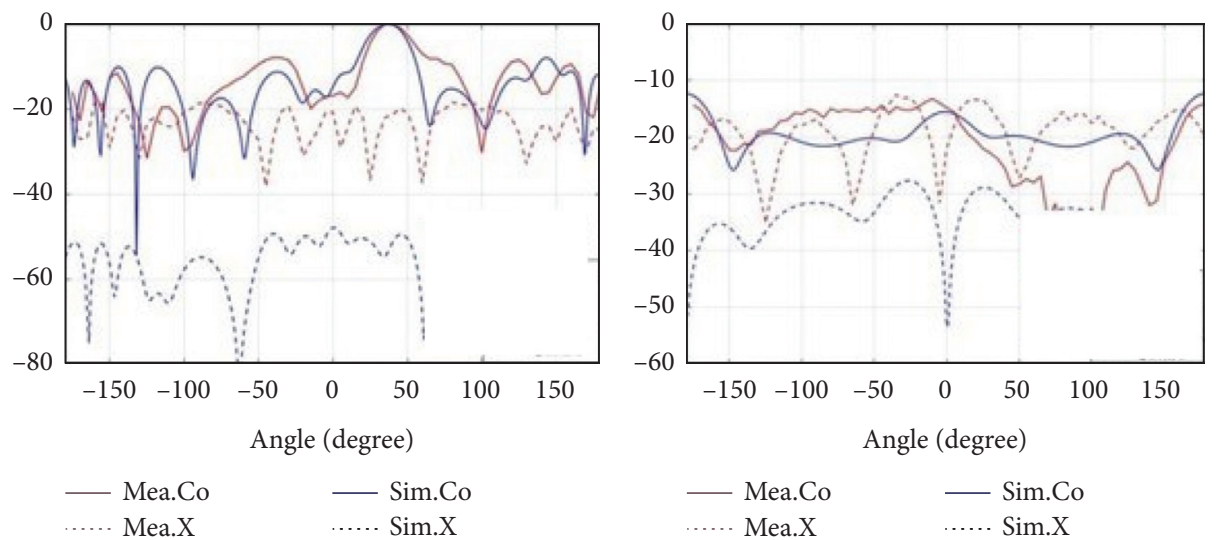

FiguRE 15: Radiation patterns at $12.0 \mathrm{GHz}, 12.5 \mathrm{GHz}$, and $12.8 \mathrm{GHz}$ with both co- and cross-polarization components; red and blue lines represent the measured and simulated results, respectively. Mea.Co and Mea.X refer to the measured co-polarization and cross-polarization components, respectively, while Sim.Co and Sim.X represent the simulated results.

between the measured and simulated results on the crosspolarization component.

In the XOY plane, the radiation beam magnitude in the broadside direction is close to that in the $Y O Z$ plane at $12.0 \mathrm{GHz}$, as the main beam direction is close to broadside at this frequency point. However, with the frequency increase, the main beam starts to tilt away from the normal direction, resulting in the fact that the maximal value in the $X O Y$ plane decreases a lot at both $12.5 \mathrm{GHz}$ and $12.8 \mathrm{GHz}$ with respect to the one in the $X O Z$ plane.

The maximal realized gain is presented in Table 2 in both simulations and measurements; this gain is almost constant over the band considered. The maximum variations are about $0.5 \mathrm{~dB}$. Also, the measured gain is about $1.5-2.0 \mathrm{~dB}$ lower than the simulated one. This is partially due to the conductor losses in the many 
TABLE 2: Realized gain and radiation efficiency at different frequency points.

\begin{tabular}{lccccc}
\hline & Freq./GHz & & 12.0 & 12.5 & \\
\hline \multirow{2}{*}{ Simu. } & Gain/dBi & 11.36 & 11.97 & 12.8 \\
& $\eta$ & $86.72 \%$ & $91.62 \%$ & \\
Meas. & Gain/dBi & 10.0 & 9.93 & 11.71 \\
& $\eta$ & $80.04 \%$ & $82.23 \%$ & 10.4 \\
& & & & $89.18 \%$
\end{tabular}

solderings, which are difficult to take into account in the simulations.

\section{Conclusion}

In this paper, the EBG units are used to generate a guiding channel on a planar board. These units are essential to ensure the transmission efficiency and eliminate the coupling effect between channels. The planar topology offers a great potential for the PCB-based practical applications and extends to design a six-element periodical leaky wave array. With a low profile $(80.6 \times 25.6 \mathrm{~mm})$, a high radiation efficiency $(86.7 \%)$, and a reasonable gain performance $(11.36 \mathrm{~dB})$ in combination with the frequency-dependent beam scanning of 30 degrees in the forward direction, this antenna topology can be applied for radar applications involving of target tracking and speed detection on road.

\section{Data Availability}

The data used to support the findings of this study are included within the article.

\section{Conflicts of Interest}

The authors declare that they have no conflicts of interest.

\section{Acknowledgments}

This work was supported in part by the China Scholarship Council under Grant 201406960007 and F. W. O. Postdoctoral Fellowship (no. 12O1217N).

\section{References}

[1] W. Volkaerts, N. V. Thienen, and P. Reynaert, "An FSK plastic waveguide communication link in 40nm CMOS," ISSCC, vol. 58, pp. 22-25, 2015.

[2] F. Distler, D. Oppelt, J. Schur, and M. Vossiek, Design and Characterization of a Compact and Robust Shielded Dielectric Waveguide for mmW Applications, pp. 375-378, GeMiC, Freiburg, Germany, 2018.

[3] G. Goubau, "Surface waves and their application to transmission lines," Journal of Applied Physics, vol. 21, no. 11, pp. 1119-1128, 1950.

[4] D. C. Lugo, R. A. Ramirez, J. Wang, and T. M. Weller, "Low permittivity cladding to improve the performance of dielectric rod waveguides and dielectric end-fire antennas," in Proceedings of the IEEE MTT-S International Microwave Symposium, San Francisco, USA, August 2016.

[5] D. D. King and S. P. Schlesinger, "Dielectric image lines," IEEE Transactions on Microwave Theory and Techniques, vol. 6, no. 7, pp. 291-299, 1958.
[6] R. M. Knox, "Dielectric waveguide Microwave integrated circuits---an overview," IEEE Transactions on Microwave Theory and Techniques, vol. 24, no. 11, pp. 806-814, 1976.

[7] P. Mondal and $\mathrm{K}$. Wu, "Single mode operation of substrate integrated non-radiative dielectric waveguide and an excitation scheme of $\$\{\backslash \mathrm{rm}$ LSE $\}$ _ 11$\} \$$ mode," IEEE Microwave and Wireless Components Letters, vol. 23, no. 8, pp. 418-420, 2013.

[8] A. Patrovsky, M. Daigle, and K. Wu, "Millimeter wave wideband transition from CPW to substrate integrated waveguide on electrically thick high-permittivity substrates," in Proceedings of the 37th EuMC, pp. 138-141, Munich, Germany, October 2007.

[9] A. Patrovsky and K. Wu, "'Substrate integrated image guide (SIIG) - a low-loss waveguide for millimeter-wave applications', in Proceedings of the 35th European. Microwave Conference, pp. 897-900, Paris, France, October 2005.

[10] Y. Cassivi and K. Wu, "NRD-guide spurious mode suppressor using self-contained periodic planar EBG structure," in Proceedings of the APMC, pp. 659-662, Taipei, Taiwan, December 2001.

[11] S. D. Assimonis, T. V. Yioultsis, and C. S. Antonopoulos, "Computational investigation and design of planar EBG structures for coupling reduction in antenna applications," IEEE Transactions on Magnetics, vol. 48, no. 2, pp. 771-774, 2012.

[12] S. F. Mahmoud and Y. M. M. Antar, "Leaky wave antennas: theory and design," in Proceedings of the Sixteenth National Radio Science Conference, pp. 1-8, Cario, Egypt, April 2013.

[13] M. Salarkaleji, M. A. Ali, and C. M. Wu, "Two-dimensional full-hemisphere frequency scanning array based on metamaterial leaky wave antennas and feed networks," in Proceedings of the IEEE International Microwave Symposium, San Francisco, USA, May 2016.

[14] W. W. Hansen, Radiating electromagnetic waveguide, 1940, https://patents.google.com/patent/US2402622A/.

[15] L. Kong, S. Yan, V. Volski, and G. A. E. Vandenbosch, "Linear nonuniform antenna array of planar elements fed by a dielectric waveguide," Microwave and Optical Technology Letters, vol. 60, no. 4, pp. 849-854, 2018.

[16] Y. J. Cheng, Y. X. Guo, X. Y. Bao, and K. B. Ng, "Millimeterwave low temperature co-fired ceramic leaky-wave antenna and array based on the substrate integrated image guide technology," IEEE Transactions on Antennas and Propagation, vol. 62, no. 2, pp. 669-676, 2014.

[17] L. Kong, S. Yan, and G. A. E. Vandenbosch, "Directive planar antenna array fed by dielectric waveguide for WiFi applications," Microwave and Optical Technology Letters, vol. 60, no. 8, pp. 1963-1967, 2018.

[18] T. Kamgaing and O. M. Ramahi, "Multiband electromagneticbandgap structures for applications in small form-factor multichip module packages," IEEE Transactions on Microwave Theory and Techniques, vol. 56, no. 10, pp. 2293-2300, 2008.

[19] D. Sievenpiper, L. Lijun Zhang, R. F. J. Broas, N. G. Alexopolous, and E. Yablonovitch, "High-impedance electromagnetic surfaces with a forbidden frequency band," IEEE Transactions on Microwave Theory and Techniques, vol. 47, no. 11, pp. 2059-2074, 1999. 
[20] Y. J. Cheng, X. Y. Bao, and Y. X. Guo, "LTCC-based substrate integrated image guide and its transition to conductor-backed coplanar waveguide," IEEE Microwave and Wireless Components Letters, vol. 23, no. 9, pp. 450-452, 2013.

[21] F. Xu and $\mathrm{K}$. Wu, "Guided-wave and leakage characteristics substrate integrated waveguide," IEEE Transactions on Microwave Theory and Techniques, vol. 53, no. 1, pp. 66-73, 2005.

[22] H. Kumar, R. Jadhav, and S. Ranade, "A review on substrate integrated waveguide and its microstrip interconnect," IOSR Journal of Electronics and Communication Engineering, vol. 3, no. 5, pp. 36-40, 2012. 